Journal for ImmunoTherapy of Cancer

\title{
PD-L1 quantification across tumor types using the reverse phase protein microarray: implications for precision medicine
}

Elisa Baldelli (D , , K Alex Hodge, ${ }^{1}$ Guido Bellezza, ${ }^{2}$ Neil J Shah, ${ }^{3}$ Guido Gambara, ${ }^{1}$ Angelo Sidoni, ${ }^{2}$ Martina Mandarano, ${ }^{2}$ Chamodya Ruhunusiri, ${ }^{1,4}$ Bryant Dunetz, ${ }^{5}$ Maysa Abu-Khalaf, ${ }^{6}$ Julia Wulfkuhle, ${ }^{1}$ Rosa I Gallagher, ${ }^{1}$ Lance Liotta, ${ }^{1}$ Johann de Bono, ${ }^{7}$ Niven Mehra, ${ }^{7}$ Ruth Riisnaes, ${ }^{7}$ Antonella Ravaggi, ${ }^{8}$ Franco Odicino, ${ }^{8}$ Maria Isabella Sereni, ${ }^{1,8}$ Matthew Blackburn, ${ }^{3}$ Angela Zupa, ${ }^{1,9}$ Giuseppina Improta, ${ }^{1,9}$ Perry Demsko, ${ }^{1}$ Lucio Crino', ${ }^{10}$ Vienna Ludovini, ${ }^{11}$ Giuseppe Giaccone, ${ }^{3}$ Emanuel F Petricoin, ${ }^{1}$ Mariaelena Pierobon (D) ${ }^{1,4}$

\section{ABSTRACT}

Background Anti-programmed cell death protein 1 and programmed cell death ligand 1 (PD-L1) agents are broadly used in first-line and second-line treatment across different tumor types. While immunohistochemistry-based assays are routinely used to assess PD-L1 expression, their clinical utility remains controversial due to the partial predictive value and lack of standardized cut-offs across antibody clones. Using a high throughput immunoassay, the reverse phase protein microarray (RPPA), coupled with a fluorescence-based detection system, this study compared the performance of six anti-PD-L1 antibody clones on 666 tumor samples. Methods PD-L1 expression was measured using five antibody clones (22C3, 28-8, CAL10, E1L3N and SP142) and the therapeutic antibody atezolizumab on 222 lung, 71 ovarian, 52 prostate and 267 breast cancers, and 54 metastatic lesions. To capture clinically relevant variables, our cohort included frozen and formalin-fixed paraffinembedded samples, surgical specimens and core needle biopsies. Pure tumor epithelia were isolated using laser capture microdissection from 602 samples. Correlation coefficients were calculated to assess concordance between antibody clones. For two independent cohorts of patients with lung cancer treated with nivolumab, RPPAbased PD-L1 measurements were examined along with response to treatment.

Results Median-center PD-L1 dynamic ranged from 0.01 to 39.37 across antibody clones. Correlation coefficients between the six antibody clones were heterogeneous (range: -0.48 to 0.95 ) and below 0.50 in $61 \%$ of the comparisons. In nivolumab-treated patients, RPPA-based measurement identified a subgroup of tumors, where low PD-L1 expression equated to lack of response.

Conclusions Continuous RPPA-based measurements capture a broad dynamic range of PD-L1 expression in human specimens and heterogeneous concordance levels between antibody clones. This high throughput immunoassay can potentially identify subgroups of tumors in which low expression of PD-L1 equates to lack of response to treatment.

\section{BACKGROUND}

Therapeutic agents targeting the programmed cell death protein 1 (PD-1) and programmed cell death ligand 1 (PD-L1) significantly increase survival in patients with cancer and are broadly used as first-line and second-line treatments across different types of solid tumors. ${ }^{1-3}$ However, the search for robust and reliable biomarkers able to predict outcome to these targeted compounds remains an open challenge in immuno-oncology. ${ }^{45}$

Tumor mutational burden has been recently approved by the Food and Drug Administration (FDA) as a predictive marker of response to pembrolizumab in unresectable or metastatic solid tumors, however its broad use still remains challenging from a technical perspective. ${ }^{6}$ Immunohistochemistry (IHC)-based assays, on the other hand, are widely used as gold-standard for assessing biomarker expression in oncology, including the FDA-approved and European ConformityMark certified companion/complementary tests for PD-L1 quantification and treatment stratification. However, the utility of these assays as companion/complementary tests in immuno-oncology remains controversial, despite their systemic use, due to their partial predictive value and lack of standardized cutoffs across antibody clones which make these assays poorly interchangeable and prone to samples' misclassification. ${ }^{7-10}$

IHC-based measurements are semiquantitative, subjective by nature, and highly dependent on antigen retrieval protocols. In addition, the colorimetric detection used in IHC-based analysis renders these platforms 
inadequate to fully capture linear relationships between expression of predictive biomarkers and outcome. Thus, the utility of the IHC may be limited as it mostly captures large scale differences across clinical samples and additional testing is usually required to correctly classify equivocal measurements. ${ }^{11}$ Quantitative assays able to provide continuous output data represent a powerful alternative from a diagnostic perspective. A recent study exploring PD-L1 expression in lung cancers using parallel reaction monitoring-based mass spectrometry (MS) showed a wide dynamic range of PD-L1 expression across samples. ${ }^{12}$ However, concordance in PD-L1 expression between continuous MS data and qualitative IHC-based measurements was weak and the broad dynamic range was only partially captured by the IHC platform. ${ }^{12}$ For a therapeutic perspective, quantitative human epidermal growth factor receptor 2 (HER2) measurements in breast cancer specimens have shown superior performance in predicting response to targeted therapies compared with IHC-based, semi-quantitative measurements. ${ }^{13-16}$ The wider dynamic range captured by these quantitative platforms has been addressed as a key element for increasing precision and for accurately distinguishing responders from non-responders, especially from IHC-negative specimens. ${ }^{1517}$

In this study we tested the feasibility of using reverse phase protein microarray (RPPA), a non-subjective, quantitative, and calibrated immunoassay currently used in precision medicine clinical trials, ${ }^{18-21}$ to capture PD-L1 expression on cancer cells and predict response to antiPD-1/PD-L1 compounds. PD-L1 expression was quantified on 64 undissected and 602 microdissected tumor epithelia with six antibody clones, including two antibodies routinely used in FDA-cleared assays (Dako 22C3 and Ventana SP142) and the therapeutic anti-PD-L1 antibody atezolizumab. Lastly, continuous PD-L1 measurements were explored along with patients' outcomes to assess the ability of the RPPA platform to identify patients that may benefit from treatment.

\section{METHODS}

\section{Sample collection}

To explore PD-L1 expression by RPPA on clinically relevant samples, we used 10 independent study sets including 666 cancer tissues (figure 1A). Sets 1-5 were collected from patients with lung cancer and included snap-frozen and formalin-fixed paraffin-embedded (FFPE) samples as well as surgical specimens and core needle biopsies. Clinical-pathological characteristics of the five cohorts of patients are presented in table 1 . Sets 1 and 2 were collected at the Santa Maria della Misericordia Hospital (Perugia, Italy) and included 33 whole tissue lysates and 58 microdissected surgical specimens, respectively. ${ }^{22}$ Matched dissected (2E) and undissected (2W) samples were collected for a subset of biospecimens included in study Set 2 for a final sample size of 73 samples. Set 3 included 45 snap-frozen Non-Small Cell Lung Cancer (NSCLC) cancers surgical specimens collected at University of Padova (Italy). ${ }^{23}$

Set 4 included pretreatment FFPE surgical specimens and core needle biopsies collected from 32 patients with lung cancer undergoing immunotherapy for which retrospective material was available for molecular analysis. Biospecimens were collected at diagnosis or during follow-up visits between 2010 and 2016 by the Lombardi Comprehensive Cancer Center at Georgetown University and the Santa Maria della Misericordia Hospital (Perugia, Italy). Matched dissected and undissected samples were available for 16 of the 32 samples. Of the 32 patients included in this analysis, 28 patients were treated with nivolumab. This group of patients was included in a subanalysis assessing the association between PD-L1 measurement by RPPA and patients' responses to treatment. Set 5 included 23 pretreatment FFPE surgical specimens and core needle biopsies collected from patients with NSCLC undergoing immunotherapy. Samples were collected at the Santa Maria della Misericordia Hospital (Perugia, Italy) between 2008 and 2015. Patients were
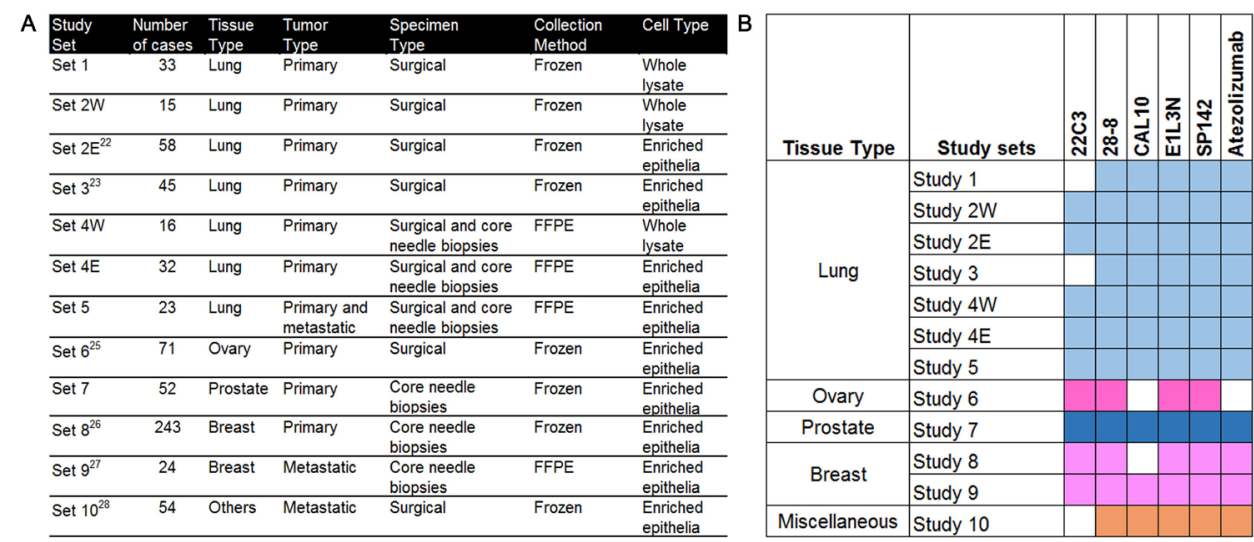

Figure 1 Main characteristics of the 10 study sets included in the analysis. A total of 666 samples were used to capture clinically relevant preanalytical variables including anatomical origin of the tumor, sample collection methods, specimen type, and cellular compartment in which programmed cell death ligand 1 (PD-L1) expression was evaluated (A). Anti-PD-L1 antibody clones used for each study set (B). E, dissected; FFPE, formalin-fixed paraffin-embedded; W, undissected. 
Table 1 Clinical pathological characteristics of patients with NSCLC included in Sets 1-5

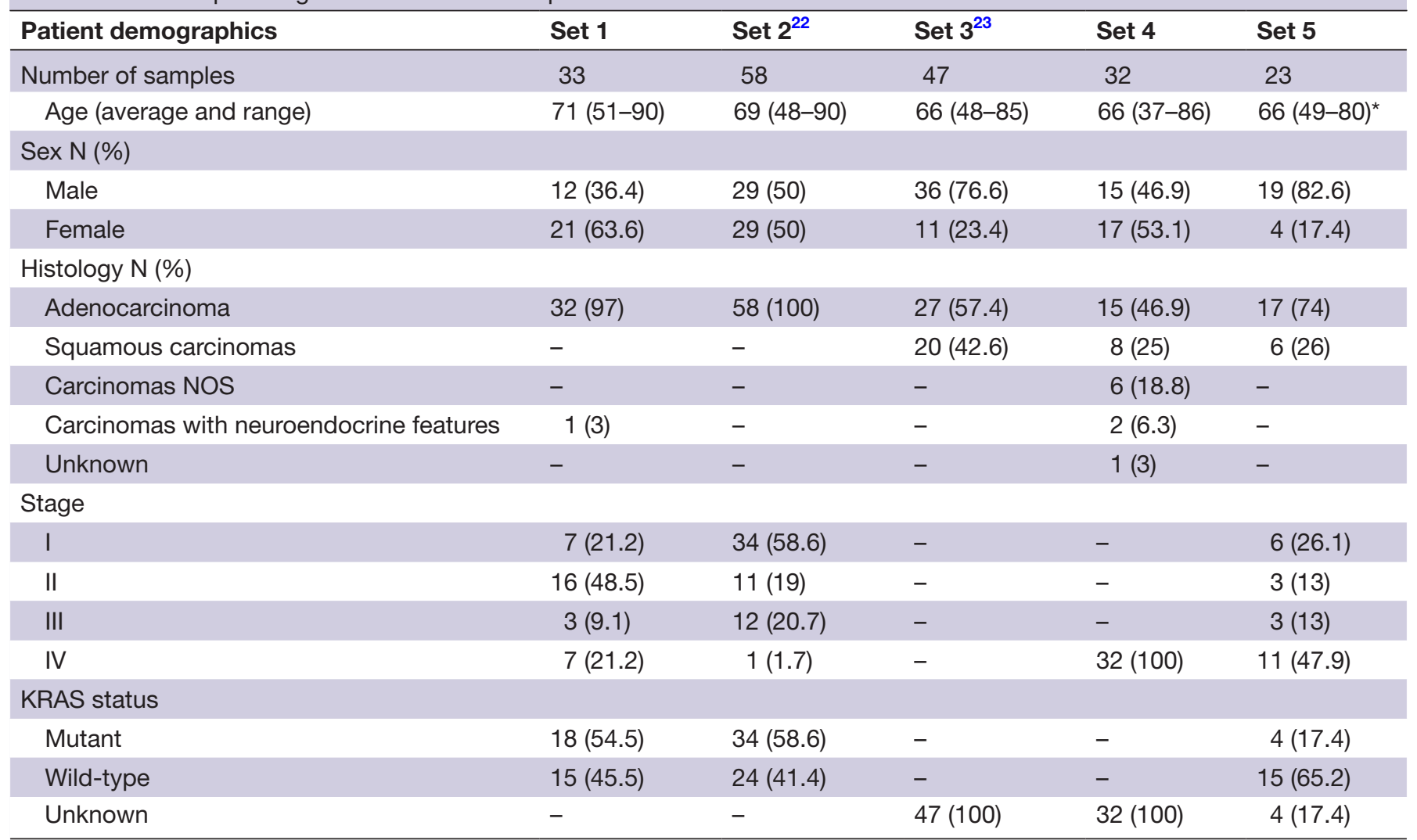

* age is missing for two patients.

KRAS, Kirsten rat sarcoma viral oncogene homolog; NOS, Not otherwise specified; NSCLC, Non-Small Cell Lung Cancer.

treated with nivolumab and response rates for Sets 4 and 5 were assessed using the Response Evaluation Criteria in Solid Tumors (RECIST) V.1.1 criteria. $^{24}$ Sets $6-10$ were used to examine the relationship between anti-PD-L1 antibody clones in different tumor types. Set 6 included 71 snap-frozen samples collected from patients with chemonaïve ovarian cancer treated at the Division of Gynecology Oncology, University of Brescia (Brescia, Italy) ${ }^{25}$ Set 7 comprised 52 snap-frozen biopsies collected from patients with prostate cancer treated at the Institute of Cancer Research (UK). Sets 8 and 9 included biospecimens collected through two clinical trials targeting patients with primary and metastatic breast cancer (NCT01042379 and NCT03195192). Specifically, Set 8 included 243 snapfrozen pretreatment core needle biopsies collected from patients enrolled in the I-SPY 2 TRIAL, ${ }^{26}$ while Set 9 included 24 FFPE pretreatment samples collected for the Side Out 3 metastatic breast clinical trial. ${ }^{27}$ Finally, Set 10 included 54 snap-frozen surgical specimens from brain metastases of breast $(n=10)$ and lung $(n=32)$ cancers, and from tumors of other origin $(n=12) .{ }^{28}$

\section{Western blotting}

The ability of the six antibodies to capture PD-L1 in denatured conditions was first tested by western blotting following standard protocols. ${ }^{29}$ Each membrane was probed with one anti-PD-L1 antibody (22C3 M3653/Dako;
28-8 ab205921/Abcam; E1L3N 13684/Cell Signaling Technology; SP142 740-4859/Roche; and atezolizumab A2004/Selleckchem) at $4^{\circ} \mathrm{C}$ overnight. Membranes were washed and subsequently incubated with a 1:10000 dilution of horseradish peroxidase-conjugated (HRP) antimouse (Invitrogen, Carlsbad, California) or anti-rabbit (Invitrogen) antibody. Atezolizumab was directly biotinylated using a commercially available kit (Lightning Link Rapid Biotin Type A, Expedeon, Heidelberg, Germany) per manufacturer's recommendations and incubated with streptavidin HRP (Invitrogen) at a 1:15000 dilution. Blots were washed and developed using a chemiluminescence HRP substrate-based system (SuperSignal West Dura, Thermo Fisher, Waltham, Massachusetts) according to the manufacturer's protocols. Images were acquired using the Eastman Kodak 4000 MM Image Station (Kodak, Rochester, New York).

\section{Laser microdissection}

For Sets 1, 2W and 4W, whole tissues were directly lysed from $8 \mu \mathrm{m}$ cryosections. Snap-frozen and FFPE tissues of Sets 2E-10 were subjected to laser microdissection. We have previously demonstrated that the laser capture microdissection (LCM) protocol described below does not affect protein expression or activation. ${ }^{30}$ For Set 4 , when possible, two cellular compartments were microdissected: the tumor epithelium and the tumor stroma 
interface (TSI), a non-cancerous area within $30 \mu \mathrm{m}$ from tumor compartment margin (online supplemental figure S1). For all 10 Sets, tissues were cut into $8 \mu \mathrm{m}$ sections and mounted onto uncharged glass slides. One Hematoxylin (Sigma Aldrich, St. Louis, Missouri) and Eosin (Sigma Aldrich) stained slide was prepared for each sample and used for histopathological evaluation. Staining protocol has been previously described. ${ }^{31}$ For Sets 4,5 and 9 FFPE sections were first deparaffinized in xylene (Sigma Aldrich) for $30 \mathrm{~min}$ and hydrated in serial dilutions of ethanol (100\%, 95\% and 70\%) (Sigma Aldrich) immediately before dissection. Frozen sections of the remaining study Sets were hydrated in $70 \%$ ethanol. All sections were then rinsed in deionized water, washed in Scotts' tap water (Electron Microscopy Sciences, Hatfield, Pennsylvania), dehydrated in ethanol $(70 \%, 95 \%$ and $100 \%)$ and xylene, and quickly dried at room temperature. From each tissue section, the tumor epithelium was collected on CapSure Macro LCM Caps (Applied Biosystems, Foster City, California).

\section{Protein extraction}

Frozen samples were lysed in a 1:1 solution of Tissue Protein Extraction Reagent (Pierce, Rockford, Illinois, USA) and 2X Tris-Glycine SDS Sample buffer (Invitrogen Life Technologies, Carlsbad, California) supplemented with $2.5 \%$ of $2 \beta$-mercaptoethanol (Sigma Aldrich). Cell lysates were boiled for $8 \mathrm{~min}$ and stored at $-80^{\circ} \mathrm{C}$. FFPE samples were lysed using the QProteome kit (Qiagen, Hilden, Germany) following the manufacturer's instructions. Briefly, polymers were removed from the CapSure Macro LCM Caps and collected into an individual screw top tube. Qiagen Extraction Buffer EXB Plus supplemented with $6 \% \beta$-mercaptoethanol was added to each screw top tube ( $1 \mu$ l of buffer per $\sim 250$ cells captured). Samples were incubated at $4^{\circ} \mathrm{C}$ for $5 \mathrm{~min}$, boiled for $20 \mathrm{~min}$ in a heating block, and subsequently placed in $80^{\circ} \mathrm{C}$ water bath for 2 hours. Afterward, samples were placed on ice for $1 \mathrm{~min}$ and centrifuged at $14000 \times \mathrm{g}$ for $15 \mathrm{~min}$ at $4^{\circ} \mathrm{C}$. Supernatants were collected and stored at $-80^{\circ} \mathrm{C}$.

\section{Reverse phase protein microarray}

Using the 2470 Aushon Arrayer (Quanterix, Billerica, Massachusetts) equipped with $185 \mu \mathrm{m}$ pins, samples were immobilized onto nitrocellulose-coated glass slides (Grace Biolabs, Bend, Oregon) in technical replicates $(n=3)$ along with a reference standard, and internal controls as previously described. ${ }^{32}$ Standard curves were prepared using commercially available cell lysates with high and low PD-L1 expression previously identified by RPPA and printed on selected arrays. SUPM2 and UV treated NIH 3T3 cells were identified as PD-L1 expressing cells and Untreated HeLa were used as control. All three cell lines were diluted to a final concentration of $0.25 \mu \mathrm{g} / \mu \mathrm{l}$. A six-point standard curve was created by mixing different amounts of the high and low expressing line to progressively decrease PD-L1 expression while maintaining a constant protein concentration across each point of the standard curve. ${ }^{33}$

A four-point bovine serum albumin serial dilution curve was printed along with the experimental samples to estimate protein amounts in each sample with a starting concentration of $1 \mu \mathrm{g} / \mu \mathrm{l}$. Protein concentration was assessed using a Sypro Ruby Protein Blot Stain (Molecular Probes, Eugene, Oregon), according to the manufacturer's instruction. In brief, arrays were fixed for $15 \mathrm{~min}$ in a $7 \%$ acetic acid and $10 \%$ methanol staining solution followed by four $5 \mathrm{~min}$ washes in deionized water and incubated with Sypro Ruby Protein Blot Stain for $30 \mathrm{~min}$.

Before immunostaining, remaining slides were treated with Reblot Plus Mild Antibody stripping solution (MilliporeSigma, Burlington, Massachusetts) for $15 \mathrm{~min}$ at room temperature, washed twice with PBS, and incubated in I-block solution (Applied Biosystems) for at least 4 hours. Immunostaining was performed on an automated system (Dako Cytomation, Carpinteria, California) where each array was probed with one antibody targeting a protein of interest. PD-L1 expression was measured with five antibody clones, including: 22C3 (1:50; mouse; M3653; Dako); 28-8 (1:500; rabbit; ab205921; Abcam); CAL10 (1:200; rabbit; AC13171A; Biocare); E1L3N (1:500; rabbit; 13684; Cell Signaling Technology) and SP142 (1:50; rabbit; 740-4859; Roche). Atezolizumab, the humanized monoclonal antibody used as a therapeutic agent targeting PD-L1, was also used as primary antibody (1:100; Human; A2004; Selleckchem). Samples were then probed with a biotinylated goat antirabbit (Vector Laboratories; 1:7500), rabbit anti-mouse (Dako; 1:10), or anti-human (Vector Laboratories; 1:7500) secondary antibodies matching the species of the primary antibody. Signal detection was performed using a commercially available tyramide-based Catalyzed Signal Amplification System (CSA, Dako) coupled with a fluorescent streptavidin-conjugated IRDye680 dye (LI-COR Biosciences, Lincoln, Nebraska) ${ }^{32}$ For each staining batch, one slide was probed with the secondary antibody only and used as negative control for normalization purposes. As a control to capture concordance between antibody clones by RPPA, three study Sets were also stained with two antibodies targeting phosphorylated S6 Ribosomal Protein (S6 Ribosomal Protein S235/236; 1:200; rabbit; 4856; Cell Signaling Technology and S6 Ribosomal Protein S240/244; 1:1000; rabbit; 2215; Cell Signaling Technology), two targeting phosphorylated AKT (AKT S473; 1:100; rabbit; 9271; Cell Signaling Technology, and AKT T308; 1:100; rabbit; 9275; Cell Signaling Technology) and two humanized antibodies targeting PD-1 (pembrolizumab; 1:100; human; A2005; Selleckchem, and nivolumab; 1:100; human; A2002; Selleckchem). To estimate immune infiltrate, the TSI was also stained with an anti-CD45 antibody (1:200; mouse; BD).

Antibody and Sypro Ruby Protein Blot stained arrays were scanned with a laser PowerScanner (TECAN, Männedorf, Switzerland) using the appropriate wavelength channel. ${ }^{32}$ Image analysis was performed using a 
commercially available software (MicroVigene V.5.1.0.0, VigeneTech). ${ }^{34}$ The software automatically performs spot finding and subtraction of local background and unspecific signal. Samples were then normalized to the amount of protein and averaged across replicates.

\section{Immunohistochemistry}

For a subset of samples in Set 4 and for Set 5, IHC-based PD-L1 expression data were collected by the pathology departments of the enrolling institutions following standard protocols. IHC data were collected using the FDAapproved 22C3 pharmDx assay for 15 of the 32 samples and with the E1L3N clone for Set 5 . IHC for clone E1L3N was performed using a laboratory developed test on FFPE archival tissues using the BOND-III fully automated IHC stainer (Leica Biosystems, Wetzlar, Germany). Antigen retrieval was performed using citrate buffer at $\mathrm{pH}=9$ for $20 \mathrm{~min}$, followed by incubation with primary antibody (1:200; rabbit; 13684; Cell Signaling Technology) for 15 min. $^{35}$

\section{Statistical analysis}

RPPA continuous data were used to assess the concordance between anti-PD-L1 antibody clones within each study Set. Given the exploratory nature of this work, to assess the ability of the RPPA platform to predict response to nivolumab, we defined low PD-L1 expressing tumors as those belonging to the bottom tertial of the population in Sets $4 \mathrm{E}$ and 5 . At large, this arbitrary cut-off value seemed to correspond with an inflection point in our sample distribution for both Sets $4 \mathrm{E}$ and 5 . Spearman rank-order correlation coefficients $\left(\mathrm{r}_{\mathrm{s}}\right)$, coefficient of determination $\left(\mathrm{R}^{2}\right)$, linear regression plots, and scatterplot matrixes were calculated and/or displayed using JMP V.5.1 (SAS Institute). Median-centered dynamic range was calculated as follows. For each population, the median value was first calculated and all samples were then normalized to the corresponding median value. Dynamic ranges were displayed using Tableau V.2020.1 (Tableau Software, Seattle, Washington). Non-parametric mean comparisons were performed in SPSS V.26. Images were modified in Photoshop V.11 (Adobe) for publication purposes.

\section{RESULTS \\ Quantitative PD-L1 measurements by RPPA on whole tissues and enriched tumor epithelia across tumor types}

Given the high analytical concordance reported in the literature, but poor cut-point reproducibility across clones resulting in misclassification of PD-L1 status for some specimens, ${ }^{7}{ }^{910}$ we first explored concordance between five diagnostic and one therapeutic anti-PD-L1 antibody clones in ten independent study Sets. A total of 666 samples were used to capture a broad range of preanalytical and clinical variables including sample collection methods (snap-frozen vs FFPE), type of input material (surgical specimens vs core needle biopsies), anatomical origin of the tumor, primary or metastatic lesions, and
PD-L1 staining on tumor cells and surrounding microenvironment (whole tissues and pure tumor epithelia) (figure 1A). Our analysis included two antibodies developed for FDA-cleared assays (Dako 22C3 and Roche SP142), ${ }^{11}$ three antibodies used in laboratory-based assays (Abcam 28-8, Cell Signaling E1L3N, Biosource CAL10), and one humanized antibody approved by the FDA as a therapeutic agent (atezolizumab) (figure 1B). The Abcam 28-8 clone targets the same epitope as the antibody included in the FDA approved IHC-based 28-8 pharmDx assay from Agilent. Five of the six antibodies were first tested using western blotting to confirm their ability to recognize epitopes in denatured lysates and assess their specificity. Bands were detected at the predicted molecular weight exclusively as previously described (online supplemental figure S2A,B). ${ }^{36}$ Affinity for the four cell lines varied from antibody to antibody. Similarly, the linear and dynamic range for each of the five diagnostic/ laboratory-developed clones against a reference standard prepared with a combination of cell lines with high and low PD-L1 expression varied greatly across antibody clones (online supplemental figure S3). ${ }^{38}$

Median-centered RPPA-based PD-L1 continuous data showed dynamic ranges between 0.01 and 39.37 across the 10 study Sets analyzed (online supplemental figure $\mathrm{S} 4,5)$. Broader distributions were detected for the CAL10 and E1L3N, with the exception of Set 6 and Set 8 where the largest dynamic range was detected for the SP142 clone. Atezolizumab had the most compressed dynamic range in 7 of the 10 (67\%) study sets. As expected, when dissected and matched undissected samples were compared, enriched epithelial samples had a broader dynamic range regardless of the sample collection method (Set 2 and Set 4 online supplemental figure S4). Pairwise comparisons between the six anti-PD-L1 antibodies showed a high level of heterogeneity, with overall low-to-moderate concordance, across the 10 study Sets. Positive correlation coefficients ranged between 0.006 and 0.95 and negative coefficients between -0.03 and -0.48 (figure $2 \mathrm{~A}-\mathrm{G}$, online supplemental figure $\mathrm{S} 6$ and table 1). The highest levels of concordance were detected between atezolizumab and the 28-8 clone in lung cancer samples where 4 of the 7 Sets had $r_{s} \geq 0.75$ (Set 1, Set 2E, Set $4 \mathrm{E}$ and $4 \mathrm{~W})$. Correlation coefficients $>0.62$ were also detected in Sets 7, 8, and 9. Generally strong correlations $\left(r_{s} \geq 0.70\right)$ were also detected between CAL10 and SP142, CAL10 and E1L3N, and E1L3N and SP142 in 6 of the 9 (66.7\%) study Sets (figure 2, online supplemental figure $\mathrm{S} 6$ and table S1). As expected, higher levels of concordance were detected in the enriched tumor epithelial samples compared with the matched whole tissue lysates (figure 2, and online supplemental figure S7).

We then compared concordance between three antibodies routinely used in FDA-cleared assays for patients' stratification to nivolumab (Abcam 28-8), pembrolizumab (Dako 22C3), and atezolizumab (Roche SP142). As expected, SP142 showed overall low level of concordance with both the 28-8 and the 22C3 clones (figure 2, 


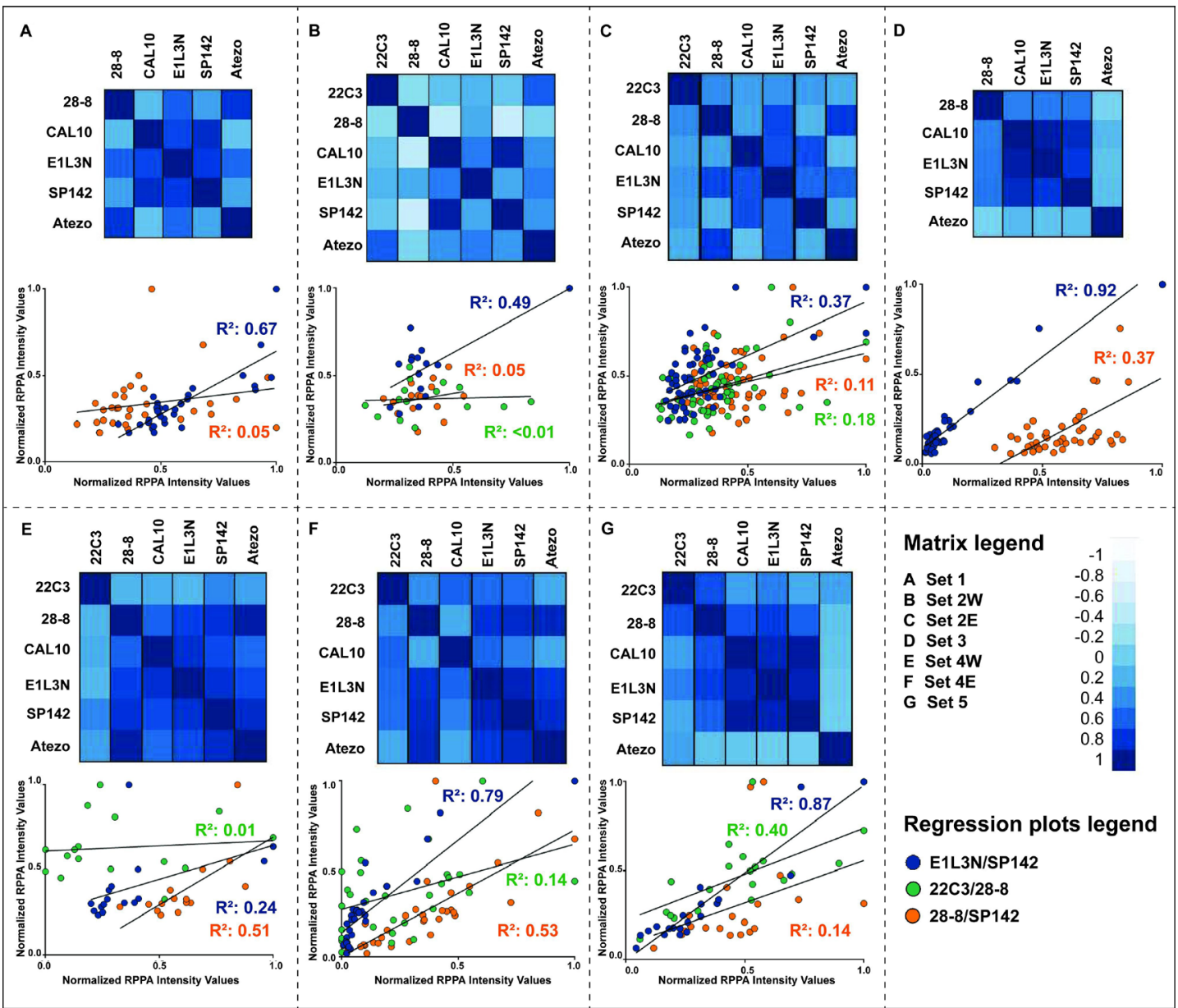

Figure 2 Correlation matrixes using Spearman's Rho correlation coefficients along with matching regression plots across anti-PD-L1 antibody clones. Levels of concordance between six anti-PD-L1 antibodies vary within the the five Non-Small Cell Lung Cancer (NSCLC) Sets. Regression plots illustrate level of concordance between antibodies used in Food and Drug Administration-cleared assays along with the research only anti-PD-L1 antibody E1L3N. E, dissected cancer epithelia; PD-L1, programmed cell death ligand 1; RPPA, reverse phase protein microarray; $\mathrm{R}^{2}$, coefficient of determination; $\mathrm{W}$, undissected whole tissue lysates.

online supplemental figure S6 and table S1). Regression analysis for SP142 and 28-8 clones showed $\mathrm{R}^{2}$ ranged between 0.01 and 0.37 with the exception of Sets $4 \mathrm{E}$ and $4 \mathrm{~W} \quad\left(\mathrm{R}^{2}>0.51\right)$ (figure 2, online supplemental figure S6). Similarly, $\mathrm{R}^{2}$ between the SP142 and the $22 \mathrm{C} 3$ clone were between 0.001 and 0.26 with the exception of Set $5\left(\mathrm{R}^{2}>0.70\right)$ (figure 2, online supplemental figure $\mathrm{S} 6$ ). On the contrary, concordance between the SP142 and E1L3N clones reached $\mathrm{R}^{2}$ between 0.79 and 0.92 for five of the analyzed sets. Finally, we explored concordance between the 22C3 and the 28-8 clones across all study Sets. $\mathrm{R}^{2}$ ranged between 0.005 and 0.18 and only Set 5 had $\mathrm{R}^{2}>0.40$ (figure 2, online supplemental figure $\mathrm{S} 6$ ). Overall these data indicate poor concordance between these three diagnostic clones regardless of tumor types, sample collection methods, and primary and metastatic lesions (figure 2A-G, online supplemental figure $\mathrm{S} 6$ and table S1).

Finally, although we did not directly isolate immune cells from our samples, for a subset of samples included in Set $4(n=26)$, we collected non-malignant cells in near proximity to the tumor cells; we called this compartment TSI. The TSI was specifically defined as the area within a $30 \mu \mathrm{m}$ distance from the tumor compartment (online supplemental figure S1). We captured PD-L1 expression within this compartment with the six anti-PD-L1 antibody clones previously described. In addition, to assess immune infiltrate in the TSI, we stained the samples with an antiCD45 antibody. We then looked at correlation levels between the different antibody clones in all 26 samples as well as in samples with high CD45 (above population median) and low CD45 (below the population median), respectively. Coefficients of determination across all samples were similar to the one detected in the tumor compartment. When samples were subclassified based on the high or low CD45 expression, coefficients of determination across antibody clones were higher in samples with a greater number of immune cells (figure 3 ).

Because correlative levels between anti-PD-L1 antibodies were lower than expected, as a control experiment, we explored concordance between antibodies targeting different activation sites of the S6 Ribosomal Protein 


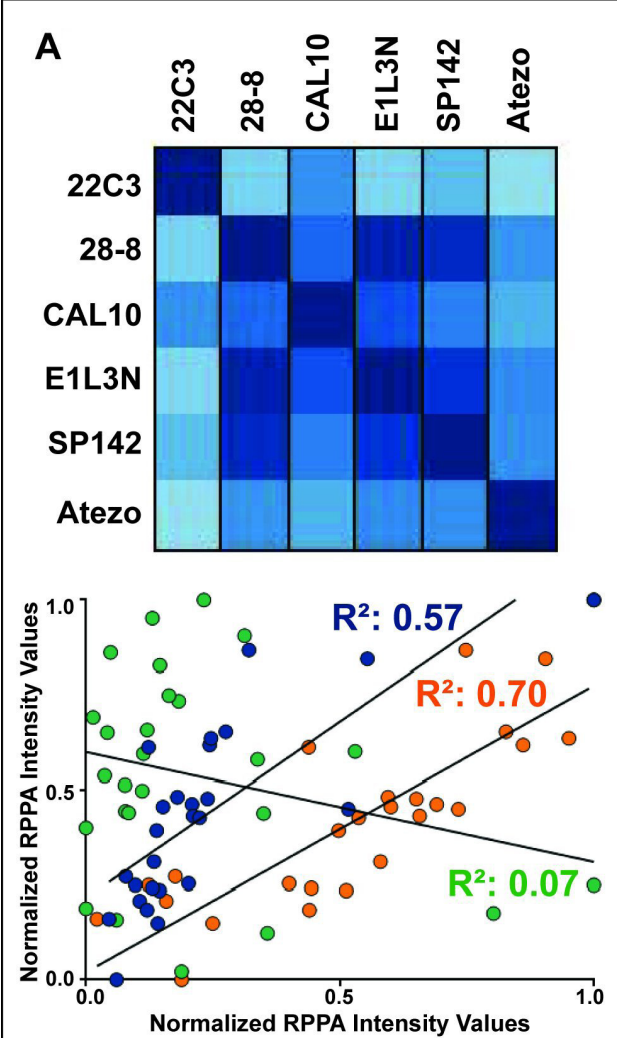

\section{Matrix legend}

A Set 4 Stroma -0.8

B CD45 High $\quad-0.6$

C CD45 Low -0.4

$-0.2$

0

0.2

0.4

0.6

0.8

1

\section{Regression plots legend}

E1L3N/SP142

22C $3 / 28-8$

28-8/SP142

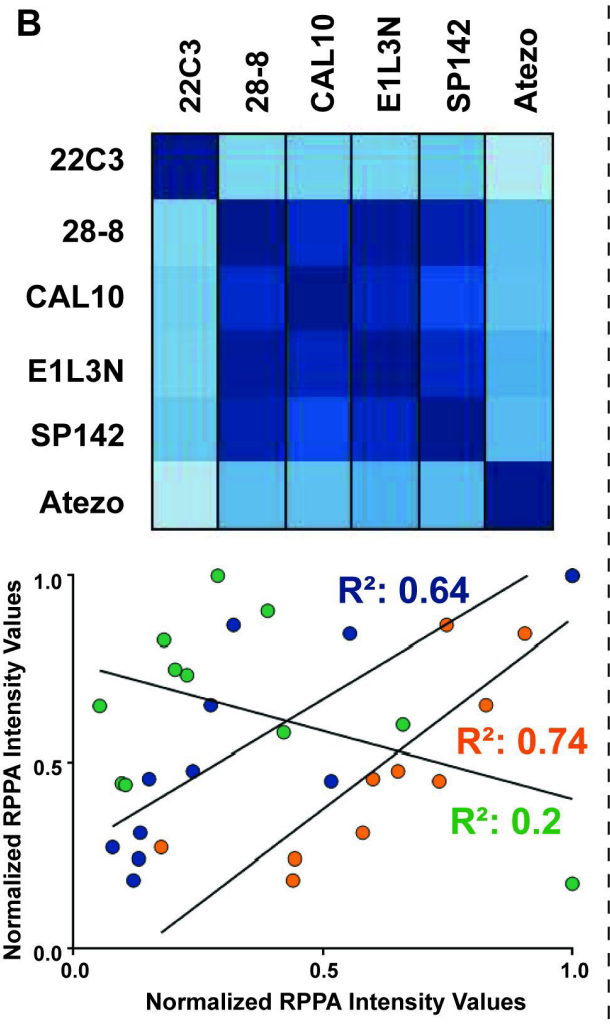

C

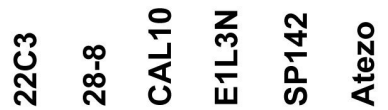

28-8

CAL10

E1L3N

SP142

Atezo
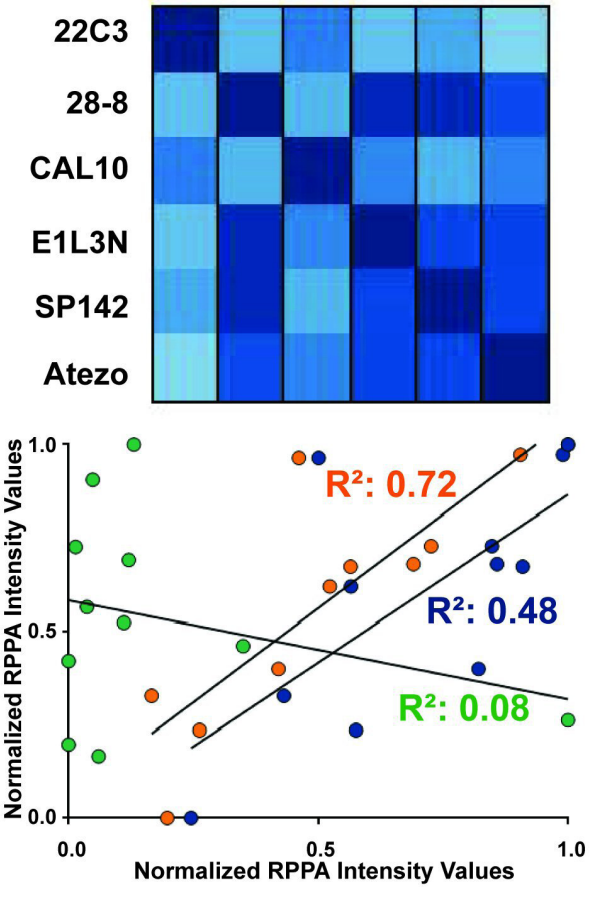

Figure 3 Correlation matrixes displaying Spearman's Rho correlation coefficients along with matching regression plots across anti-PD-L1 antibody clones in the tumor-stroma interface. Levels of concordance between six anti-PD-L1 antibodies in the tumor stroma interface of samples included in Set 4 are shown across all samples (A) and in samples with high CD45 (B) and low CD45 expression (C). Regression plots illustrate levels of concordance between antibodies used in Food and Drug Administration-cleared assays along with the research only anti-PD-L1 antibody E1L3N. PD-L1, programmed cell death ligand 1 ; RPPA, reverse phase protein microarray; $R^{2}$, coefficient of determination. 
and AKT in 3 of the 10 study Sets. As shown in online supplemental figure S8A,B, concordance levels between antibodies targeting activation sites of the S6 Ribosomal Protein were $>0.90$. Similar values were also obtained for the activated AKT regardless of tissue of origin, collection methods, and dissection process (range $r_{s}=0.70$ to 0.96). Likewise, therapeutic antibodies targeting the PD-1 receptor, namely pembrolizumab and nivolumab, showed correlation coefficients ranging from 0.83 to 0.93 (online supplemental figure S8C). Taken together, these data suggest a lower level of concordance across different anti-PD-L1 antibody clones than what has been reported for IHC-based quantification $\left(r_{s}<0.50\right.$ for $61 \%$ of pairwise comparisons).

\section{Quantitative PD-L1 measurements as predictor of response in patients with lung cancer treated with nivolumab}

Given the heterogeneous and highly dynamic range of PD-L1 expression captured by RPPA, continuous PD-L1 measurements were explored along with patients' outcomes to assess the ability of the platform to identify patients that may benefit from treatment. For this analysis we used 28 samples included in Set 4 and 23 samples included in Set 5. Retrospective cohorts of samples were collected from patients with lung cancer undergoing treatment with nivolumab. Based on RECIST V.1.1 criteria, ${ }^{24} 11$ of the 28 patients $(39.3 \%)$ in Set 4 and 9 of the $23(39.1 \%)$ in Set 5 benefited from nivolumab-based treatment (partial response and stable disease) (online supplemental table S2).

First, we captured concordance between IHC-based measurements and RPPA data for a subgroup of these 28 patients in Set 4 (figure 4A). IHC data were collected by the enrolling institutions using clone 22C3 $(n=15)$ and scored by a certified pathologist (MM, GB for samples collected in Italy). Samples scored as negative by IHC presented a sevenfold dynamic range in PD-L1 expression by RPPA. Of interest, only one of the seven responders was correctly identified by IHC using the 22C3 clone (figure 4A,D). In addition, three of the responders $(60 \%)$ classified as negative by IHC presented with PD-L1 expression levels by RPPA comparable to IHC positive individuals. These false negative results may be associated with tissue fixation, antibody dilution, and epitope retrieval methods required for the IHC analysis. ${ }^{39}$ Thus, while the level of concordance between the two techniques was relatively low, the RPPA correctly defined a subgroup of responders $(n=3)$ that were missed by IHC. A few IHC representative images of responders and nonresponders are shown in figure $4 \mathrm{D}$ along with matched PD-L1 measurements by RPPA.

We then examined PD-L1 expression in Set 4 using the 28-8 clone provided by Abcam, which was developed against the same peptide sequence used for the antibody included in the 28-8 PharmDX assay. This assay has been approved by the FDA as a complementary test for patients stratification to nivolumab. As expected, PD-L1 expression was highly heterogeneous across responders and non-responders (online supplemental figure S9). However, a subgroup of non-responders (53\%) presented with PD-L1 levels significantly lower than any of the patients that benefited from treatment $(\mathrm{p}<0.01)$ (figure 5). Among the nine non-responders with low PD-L1 expression by RPPA, equal to the bottom tertial of the population, eight out of nine $(88.8 \%)$ were also correctly identified by atezolizumab and seven out of nine $(77.8 \%)$ by the FDA-approved clone SP142 and the research-based clone E1L3N (figure 5).

Similar trends were also observed for Set 5 where PD-L1 expression was captured by IHC and RPPA using the E1L3N clone (figure 4B). Overall, samples with high PD-L1 levels by IHC presented with high relative expression by RPPA, suggesting an overall good level of concordance especially in tumors with high expression of PD-L1. However, a number of IHC negative samples $(n=6)$ had PD-L1 levels by RPPA that mimic the IHC positive specimens (figure 4B). Of interest, within IHC negative specimens, the RPPA-based PD-L1 measurements identified a subgroup of patients $(n=5$ red arrow in figure $4 B)$ that benefited from nivolumab. As shown for Set 4, six of the seven samples $(85.7 \%)$ belonging to the bottom tertial of the population did not benefit from treatment with nivolumab. The data presented in Set $4 \mathrm{E}$ and 5 suggest that lack of response to nivolumab in patients with low PD-L1 expressing tumors, may be more correctly predicted by RPPA than by standard IHC. Thus, RPPA-based measurements may be a valuable tool for identifying subgroups of specimens that may be misclassified by standard IHC as low PD-L1 expressing tumors (figure 6). Taken together, our data indicate that RPPA-based measurements may offer new opportunities for developing diagnostic tools for stratifying patients to immunotherapy.

\section{DISCUSSION}

Undoubtedly, immune-checkpoint inhibitors are a highly beneficial class of compounds for patients with cancer. ${ }^{1}$ However, the development of accurate molecular assays and the identification of biomarkers to effectively stratify patients to treatment remains an unmet need in oncology. ${ }^{40}$ FDA approved IHC-based companion/ complementary assays for the detection of PD-L1 have shown broad sensitivity and specificity in NSCLC (36\%$84 \%$ and $48 \%-78 \%$, respectively). ${ }^{42}$ While replacing IHC scoring as a routine clinical practice remains unfeasible, devising novel orthogonal tests, like tumor mutational burden testing, that can be used alongside with standard IHC assays, remains an important aspect for improving the diagnostic process and response prediction to antibodybased immunotherapies. For example, the LCM/RPPA workflow described, which has now graduated to a commercially available Clinical Laboratory Improvement Amendments/Laboratory Developed Test (CLIA/LDT) assay, may be used alongside with conventional IHC testing to allocate patients to treatment (figure 6). The standardized format of the assay is designed to capture molecular 


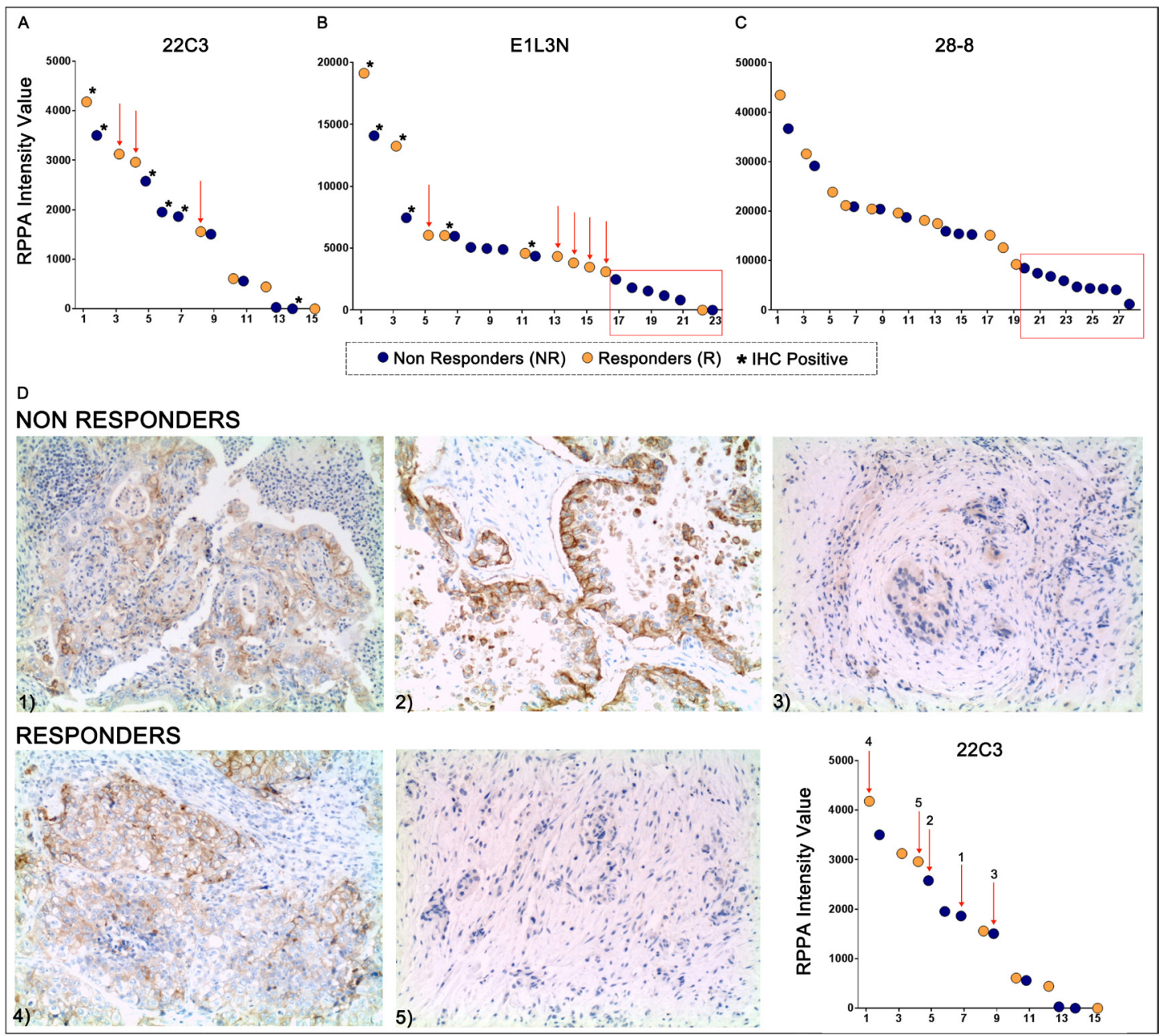

Figure 4 RPPA and matched IHC-based PD-L1 expression comparison in tissues collected from nivolumab treatment patients with Non-Small Cell Lung Cancer (NSCLC). Ranking plots capturing matched RPPA and IHC PD-L1 expression data for a subset of samples collected from responders (orange) and non-responders (blue) for Set 4 and Set 5 (A and B). PD-L1 expression was measured using the anti-PD-L1 clones 22C3 and E1L3N, respectively. RPPA continuous values are displayed on the $\mathrm{x}$-axis and asterisks indicate IHC positive samples. Red arrows indicate $\mathrm{IHC}$ negative samples that presented with relatively high expression level of PD-L1 by RPPA and responded to treatment. Ranking plots for Sets 4 and 5 (B and C) indicate that samples within the bottom tertial of the population (red box) were mostly collected from patients that did not benefit from treatment. IHC images of selected tissue samples collected from non-responders (Samples 1-3) and responders (Samples 4-5) to nivolumab (20x magnification). Of note, although defined as positive by IHC, sample 1 intensity staining was scored as 1 in $10 \%$ of cells. IHC were scored by a certified pathologist (GB and MM for samples collected at the University of Perugia). Corresponding RPPA values are highlighted in the scatter plot (bottom right corner). The RPPA measurements identified two specimens from non-responders ( 1 and 3 ) with relatively low PD-L1 expression compared to IHC positive tumors; both specimens derived from responders ( 4 and 5 ) had high PD-L1 levels by RPPA including a sample that was scored as negative by IHC (5). These data only partially matched the IHC scoring (D). IHC, immunohistochemistry; PD-L1, programmed cell death ligand 1; RPPA, reverse phase protein microarray.

data from individual or batched samples and the staining procedure mimics many of the steps used for IHC-based analyses (figure 7). Thus, the assay can be performed on a daily basis and capture expression of target analytes for batched or individual samples. As we have previously demonstrated, the entire LCM/RPPA workflow described can generate results for small panels of biomarkers in a short turnaround time ( 3 business days), with a minimal impact on treatment initiation. ${ }^{18}$ Given the potential of the RPPA to provide expanded dynamic range of PD-L1 expression, if validated on a larger study set, this assay may help identify, among patients whose tumors have low or negative PD-L1 expression by IHC, those that are destined to benefit from anti-PD-L1/PD-1 treatment.

From a technical perspective, as a high throughput and multiplex assay able to generate continuous data, the RPPA represents a unique and complementary tool to standard IHC (figure 6). Because denatured cell/tissue lysates are directly immobilized onto nitrocellulose slides, the RPPA allows for the quantification of membrane, cytosolic, and nuclear proteins without requiring tissue permeabilization or antigen retrieval, two steps that are crucial and have a strong impact on IHC-based measurements. ${ }^{39344}$ In addition, the fluorescence-based detection 


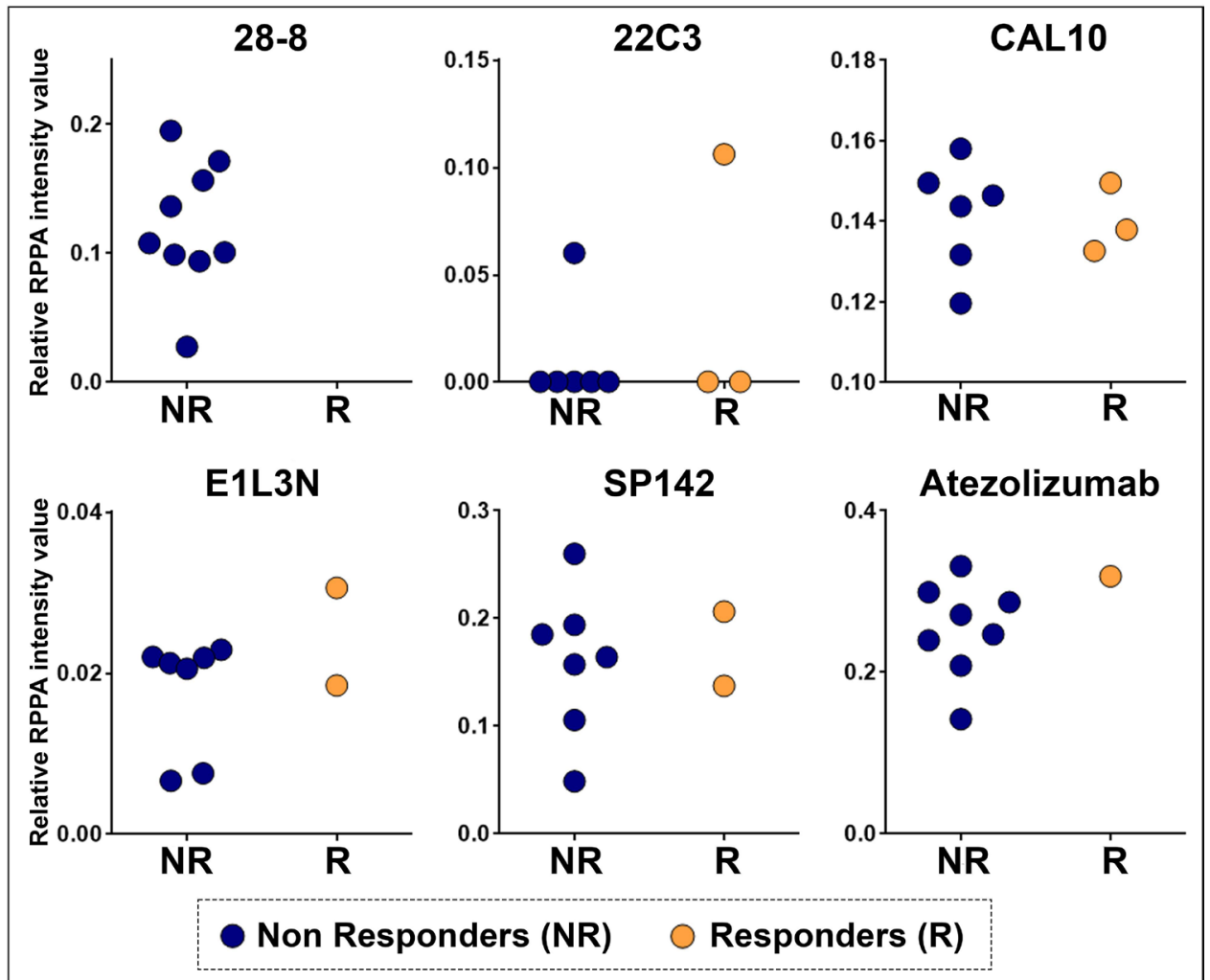

Figure 5 RPPA-based PD-L1 expression captured with six anti-PD-L1 antibody clones in patients with lung cancer treated with nivolumab. Normalized relative RPPA intensity units are displayed to capture PD-L1 distribution across the 28 samples collected from nivolumab treated patients based on patients' outcome. Emphasis is placed on the bottom tertial of the population $(n=9)$. PD-L1, programmed cell death ligand 1; RPPA, reverse phase protein microarray.

system used for the RPPA has important advantages over the chromogenic substrates routinely used in IHC-based analyses. For example, the biophysical properties and absorbance of the 3,3'-Diaminobenzidine affect signal to noise ratio and the analytical dynamic range of detection of the assay. ${ }^{11} 14$ As such, output data for FDA approved companion/complementary tests remain qualitative in nature and fail to capture the linear relationship between biomarker expression levels and outcome. ${ }^{11}$ Quantitative fluorescence-based assays have previously been tested to explore range of expression and prediction of response to targeted agents and have shown superior predictive value compared with standardized IHC, especially for biospecimens within the lower range of detection of the assay. ${ }^{13-16}$ As shown by our data, the use of a fluorescencebased detection system allows capture of broad dynamic ranges of protein expression on a continuous scale even in samples that are defined as negative by conventional IHC testing.

Of interest, our data also suggest a more heterogeneous and often a lower degree of correlation across anti-PD-L1 antibody clones compared with IHC-based

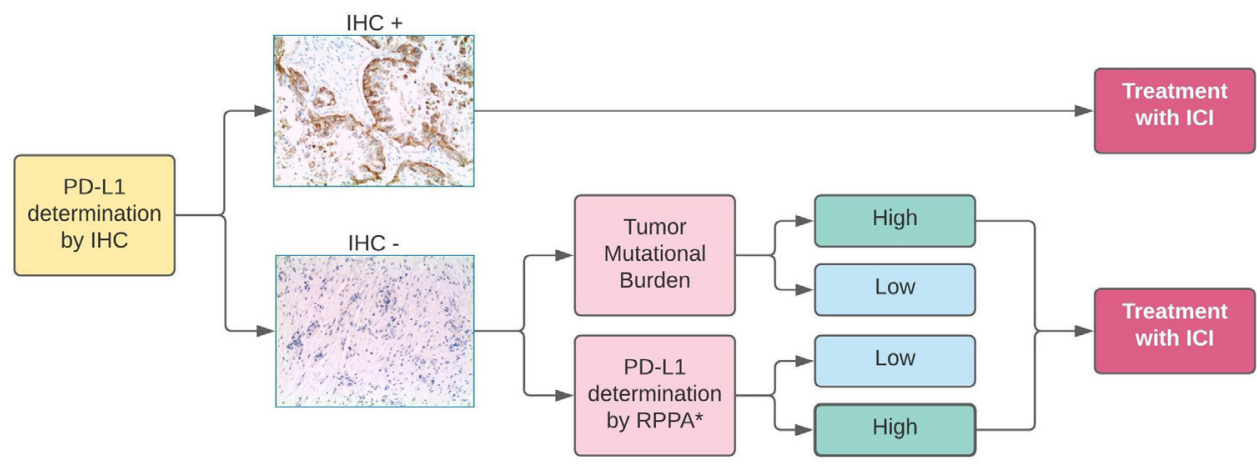

${ }^{*}$ Results delivered in 3 business days.

Figure 6 Workflow illustrating the potential role of the RPPA in allocating patients to anti-PD-1/PD-L1 treatment. PD-L1 expression on microdissected tumor cells by RPPA can be used alongside tumor mutational border to identify patients with low PD-L1 expression levels by IHC that may benefit from anti-PD-1/PD-L1 treatment. IHC, immunohistochemistry; PD-L1, programmed cell death ligand 1; RPPA, reverse phase protein microarray. 

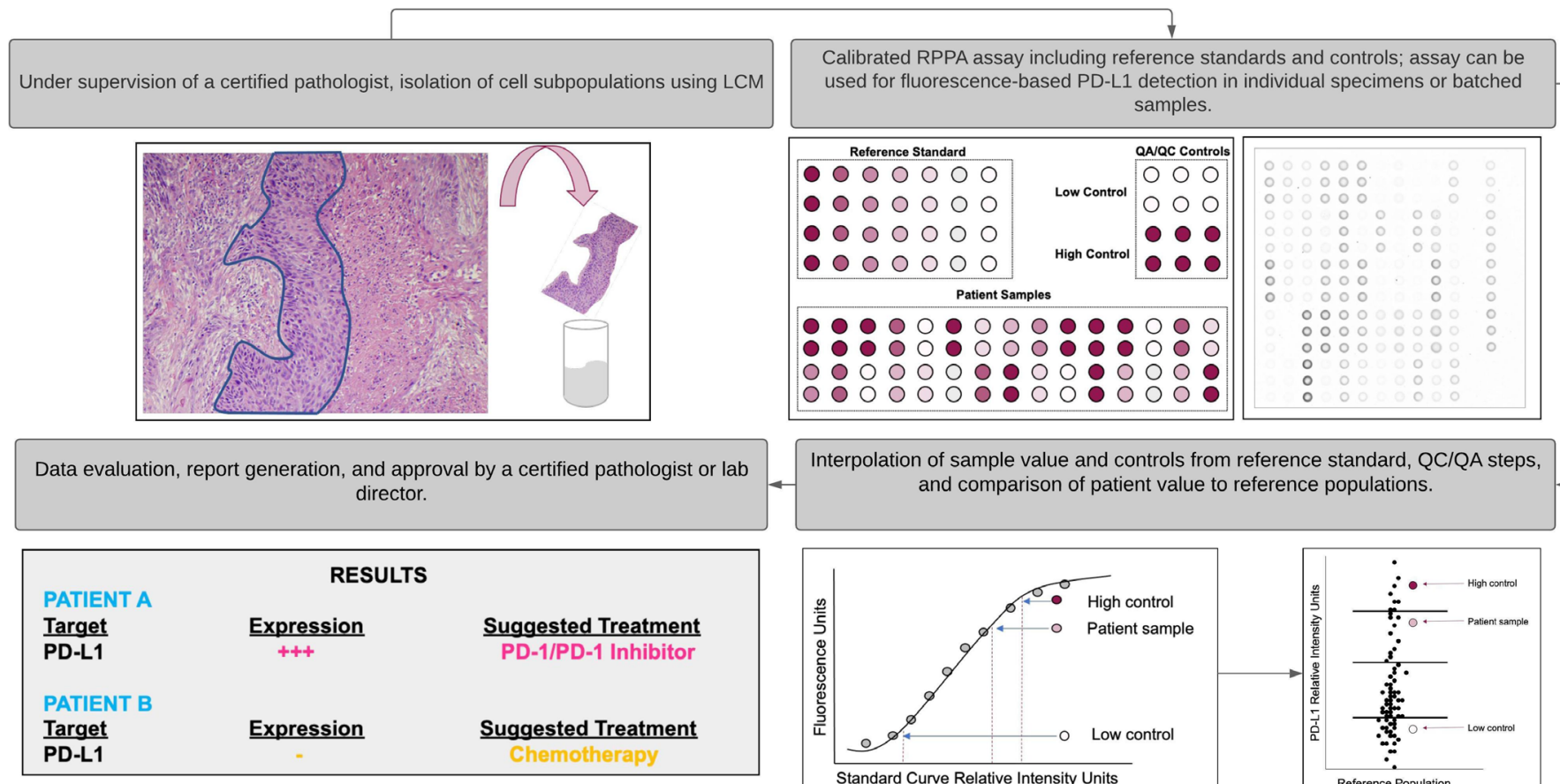

Interpolation of sample value and controls from reference standard, QC/QA steps, and comparison of patient value to reference populations.

Figure 7 Workflow illustrating the use of LCM coupled with RPPA in the diagnostic setting. First, tumor content in surgical samples and core needle biopsies is assessed by a certified pathologist and malignant cells are isolated from the surrounding microenvironment using LCM (A). Isolated cells are then lysed and immobilized onto nitrocellulose coated glass slides using a robotic system. Reference standard spanning the dynamic range of the analyte of interest and a set of internal controls are printed alongside with the clinical samples. Arrays are stained using an antibody-based detection system and absolute intensity values are generated for each sample and control (B). Intensity values of individual samples and controls are then interpolated from the reference standard and compared with a reference population matching the clinical characteristics of the samples (C). Expression levels of the measured analysis in the control samples are used as QA/QC steps to track precision and accuracy of the assay $(\mathrm{C})$. Final results and QA/QC data are reviewed by a certified pathologist or a laboratory director and included in a final report (D). LCM, laser capture microdissection; PD-1, programmed cell death protein 1; PD-L1, programmed cell death ligand 1; RPPA, reverse phase protein microarray.

comparisons, $79103845-49$ although levels of concordance differ significantly across studies. ${ }^{38} 5051$ Methodological and biological reasons may explain the differences in performance across antibody clones. For example, low levels of concordance between the 22C3 anti-PD-L1 antibody and the remaining clones may be due to unspecific binding of the 22C3 antibody to cytosolic proteins as shown in online supplemental figure S10. In addition, location of the target epitopes may explain the divergent measurements captured by RPPA. Indeed, the SP142, CAL10 and E1L3N clones bind to the intracellular portion of PD-L1. ${ }^{37}$ The recognition of cytosolic portions of the ligand by these three antibodies may explain the relatively higher level of concordance detected by RPPA between the SP142, CAL10, and E1L3N antibodies. The 22C3 and 28-8 clones, on the other hand, recognize distinct fragments of the extracellular portion of PD-L1, with the 22C3 binding to non-linear epitopes and the 28-8 clone recognizing discontinuous segments of the extracellular domains. ${ }^{52}$

Similarly, quantitative immunofluorescence-based HER2 measurements in breast cancer specimens using antibodies targeting intracellular and extracellular domains of the receptor have shown discordant levels of HER2 expression. These differences are lost in IHC-based analyses. However, only HER2 quantification captured by the antibody targeting the extracellular portion of the receptor was associated with response to trastuzumab. ${ }^{14}$ Cleavage of the extracellular portion of the HER2 and expression of truncated forms of the receptor were identified as potential biological mechanisms responsible for these discrepancies. Likewise, the extracellular domain of PD-L1 is a known target of extracellular matrix metalloproteases. $^{25354}$ This may explain the lower correlation levels detected by RPPA across antibody clones and the ability of the 28-8 clone to identify a subgroup of tumors on the lower end of the detection range that did not benefit from treatment (figure 4).

Indeed, when association between PD-L1 levels and response to treatment with nivolumab was analyzed, the RPPA continuous measurements identified a subgroup of non-responders $(53 \%)$, equal to the bottom tertial of the population distribution, with PD-L1 levels lower than any of the patients that experienced partial response or stable disease. Retrospective biomarker analyses of phase III clinical trials assessing the predictive value of PD-L1 expression by IHC in second-line treatment with nivolumab have reported response rates of approximately $20 \%$ for patients with PD-L1 positive lung cancer. ${ }^{4}$ In the pretreated cohort of patients included in this 
analysis, benefit from nivolumab was observed in 11 of the 19 tumors $(\sim 60 \%)$ with PD-L1 levels greater than the bottom tertial of the population. While IHC scoring with the 28-8 clone is not currently required to allocate patients to treatment with nivolumab, and any level of PD-L1 expression is considered a potential indicator of response, identifying predictive markers of response to nivolumab-based treatment still remains an open challenge in immuno-oncology. Even if very preliminary, by identifying a subgroup of tumors with low PD-L1 expression that do not respond to treatment, our data indicate that PD-L1 expression captured by RPPA with the FDAapproved 22-8 clone may provide valuable insights to the diagnostic process. However, because the cut-off value used in this work was arbitrary, our findings need to be validated on a larger cohort of samples after the assay has been standardized. ${ }^{33}$

Although outside of the scope of this work, the standardization of this assay can easily be developed as previously described (figure 7) ${ }^{21335556}$ First, PD-L1 expression levels measured by RPPA need to be collected from a large cohort of patients with NSCLC treated with a PD-L1/ PD-1 inhibitor (training cohort) along with reference standards. This process is a key step for assessing whether the dynamic range of the population is contained within the linear range of the reference standards and for identifying a cut-off value able to discriminate responders from non-responders (online supplemental figure S11). Controls with values above and below the cut-off are then identified and used to assess the performance of the platform over time. A second independent cohort of samples (validation cohort) is then used to assess the ability of the test to predict response to treatment. For each sample in the validation cohort, absolute RPPA values are transformed into relative intensity values of the reference standard and compared with the reference population to predict response to treatment (figure 7). If the assay shows sensitivity, specificity, and positive/negative predictive values that are equal or superior to other FDA-approved tests, the assay can then be used to analyze individual or batched samples (figure 7).

While our preliminary data are encouraging, a few limitations of the study need to be addressed. First, the clinical relevance of our observations was assessed in two small cohorts of samples. Thus, to fully understand the role of the RPPA in predicting response to treatment with anti-PD-1/PD-L1 inhibitors, the assay needs to be standardized and tested against larger and independent sample sets as described above (figure 7). Second, although we have previously demonstrated that the LCM/ RPPA workflow can be used to analyze clinical trials specimens, ${ }^{20335556}$ the routine integration of the LCM into clinical and molecular pathology may be challenging from a technical perspective as it adds time and effort to the diagnostic process. However, LCM and microdissection techniques in general are a growing component of a number of clinical Next Generation Sequencing (NGS) workflows and have recently been assigned Current Procedural
Terminology (CPT) codes (ie, 88380 and 88381). Thus, integration of these enrichment methods in clinical practice may increase in the near future. Lastly, the presence of preanalytical variables represent a potential bias of this work. However, trends were highly consistent across data sets. For example, correlation between antibodies used in FDA-cleared assays showed overall low levels of concordance in all data sets with all but two presenting with $r_{s}$ of less than 0.60 when correlations between the SP142 and 28-8 clones were analyzed. Similar values were also captured for the correlations between the 28-8 and the $22 \mathrm{C} 3$ clones $(\mathrm{r}<0.60$ in all but one Set). On the contrary, we detected higher levels of concordance across the board with the remaining clones. For example, correlations between CAL10 and SP142, CAL10 and E1L3N, or E1L3N and SP142 had $r_{s}$ greater than 0.7 in 9 of the 10 $(90 \%)$ study Sets. This high level of concordance across data sets provides an analytical validation of our observations and indicates that most likely preanalytical variables are not driving our observations.

Taken together, our data indicate that continuous RPPAbased measurements capture a broad dynamic range of PD-L1 expression in human specimens. Although this data needs to be validated in larger and independent cohorts of samples, using both diagnostic and therapeutic anti-PD-L1 antibodies, this high throughput immunoassay can potentially identify, especially on the lower end of the PD-L1 expression distribution, a subgroup of tumors in which low expression of the ligand equates to lack of response to anti-PD-1 agents. As previously shown, RPPA standardized measurements can easily be generated from laser captured microdissected clinical trial specimens, including small core needle biopsies, and can be reported to treating physicians within $3-10$ business days from sample collection. ${ }^{18} 1933$ Thus, this platform may open novel opportunities for identifying patients that may benefit from treatment with anti-PD-1/PD-L1 agents as well as other immune-checkpoints inhibitors. As such, the predictive value of RPPA-based PD-L1 measurements and of the different antibody clones should be tested in future retrospective and prospective investigations to validate its role as a companion test for allocating patients to treatment.

\section{Author affiliations}

${ }^{1}$ Center for Applied Proteomics and Molecular Medicine, George Mason University, Manassas, Virginia, USA

${ }^{2}$ Department of Experimental Medicine, Section of Anatomic Pathology and Histology, University of Perugia, Perugia, Italy

${ }^{3}$ Department of Oncology, Lombardi Comprehensive Cancer Center, Georgetown University, Washington, District of Columbia, USA

${ }^{4}$ School of Systems Biology, George Mason University, Manassas, Virginia, USA ${ }^{5}$ Side Out Foundation, Fairfax, Virginia, USA

${ }^{6}$ Department of Medical Oncology, Sidney Kimmel Cancer Center at Jefferson Health, Thomas Jefferson University, Philadelphia, Pennsylvania, USA

${ }^{7}$ The Institute of Cancer Research, London, UK

${ }^{8}$ Angelo Nocivelli Institute of Molecular Medicine, Division of Gynecologic Oncology, University of Brescia and ASST Spedali Civili di Brescia, Brescia, Italy

'Unita' Operativa di Anatomia Patologica, Istituto di Ricovero e Cura a Carattere Scientifico (IRCCS) CROB, Rionero In Vulture, Italy 
${ }^{10}$ Department of Medical Oncology, Istituto Scientifico Romagnolo per lo Studio e la Cura dei Tumori (IRST) IRCCS, Meldola, Italy

${ }^{11}$ Division of Medical Oncology, S. Maria della Misericordia Hospital, Perugia, Italy

\section{Twitter Martina Mandarano @MartinaMandara4}

Contributors Conceptualiastion/study design: EB, EFP, MP. Sample and data collection: LC, VL, GGa, GGi, NJS, MB, AR, FO, RR, JdB, AS, BD, MA-K, NM. Experimental procedures: EB, GB, GGa, CR, MM, JW, RIG, LL, MIS, AZ, GI, PD, MP. Data analysis and interpretation: EB, GB, MM, AS, EFP, MP. Manuscript preparation and final approval: All authors.

Funding This work was supported by the Side Out Fondation, the Istituto Superiore di Sanità (Rome, Italy), and by the College of Science, George Mason University. The I-SPY work was conducted with support from Quantum Leap Healthcare Collaborative, FNIH, NCI (Grant P01 CA210961), Safeway, an Albertsons Company, William K. Bowes, Jr. Foundation, Breast Cancer Research Foundation, UCSF, GMU, Gateway for Cancer Research (Grant G-16-900), the Biomarkers Consortium, Salesforce, OpenClinica, Formedix, Natera, Hologic, TGen, Illumina, CCS Associates, Berry Consultants, Breast Cancer Research - Atwater Trust, Stand up to Cancer, California Breast Cancer Research Program, and Give Breast Cancer the Boot, IQVIA, Genentech, Amgen, Pfizer, Merck, Seattle Genetics, Daiichi Sankyo, AstraZeneca, Dynavax Technologies, Puma Biotechnology, AbbVie, Madrigal Pharmaceuticals (formerly Synta Pharmaceuticals), Plexxikon, Regeneron, and Agendia.

Competing interests The authors are inventors on US Government and University assigned patents and patent applications that cover aspects of the technologies discussed such as the Reverse Phase Protein Microarrays. As inventors, they are entitled to receive royalties as provided by US Law and George Mason University policy. MP, JW, LL and EFP receive royalties from and are consultants of TheraLink Technologies. EFP and LL are shareholders and consultants of TheraLink Technologies. EFP is shareholder and consultant of Perthera.

\section{Patient consent for publication Not applicable.}

Ethics approval Sample collection and their use for molecular analysis were approved by the enrolling institutions. Samples were collected under voluntary consent.

Provenance and peer review Not commissioned; externally peer reviewed. Data availability statement Data are available upon reasonable request.

Supplemental material This content has been supplied by the author(s). It has not been vetted by BMJ Publishing Group Limited (BMJ) and may not have been peer-reviewed. Any opinions or recommendations discussed are solely those of the author(s) and are not endorsed by BMJ. BMJ disclaims all liability and responsibility arising from any reliance placed on the content. Where the content includes any translated material, BMJ does not warrant the accuracy and reliability of the translations (including but not limited to local regulations, clinical guidelines, terminology, drug names and drug dosages), and is not responsible for any error and/or omissions arising from translation and adaptation or otherwise.

Open access This is an open access article distributed in accordance with the Creative Commons Attribution Non Commercial (CC BY-NC 4.0) license, which permits others to distribute, remix, adapt, build upon this work non-commercially, and license their derivative works on different terms, provided the original work is properly cited, appropriate credit is given, any changes made indicated, and the use is non-commercial. See http://creativecommons.org/licenses/by-nc/4.0/.

\section{ORCID iDs}

Elisa Baldelli http://orcid.org/0000-0002-6288-4599

Mariaelena Pierobon http://orcid.org/0000-0003-2084-1029

\section{REFERENCES}

1 Akinleye A, Rasool Z. Immune checkpoint inhibitors of PD-L1 as cancer therapeutics. J Hematol Oncol 2019;12:92.

2 Gong B, Kiyotani K, Sakata S, et al. Secreted PD-L1 variants mediate resistance to $P D-L 1$ blockade therapy in non-small cell lung cancer. $J$ Exp Med 2019;216:982-1000.

3 Carretero-González A, Lora D, Ghanem I, et al. Analysis of response rate with ANTI PD1/PD-L1 monoclonal antibodies in advanced solid tumors: a meta-analysis of randomized clinical trials. Oncotarget 2018;9:8706-15

4 Teixidó C, Vilariño N, Reyes R, et al. PD-L1 expression testing in non-small cell lung cancer. Ther Adv Med Oncol 2018;10:1758835918763493.
5 Havel JJ, Chowell D, Chan TA. The evolving landscape of biomarkers for checkpoint inhibitor immunotherapy. Nat Rev Cancer 2019;19:133-50.

6 Chan TA, Yarchoan M, Jaffee E, et al. Development of tumor mutation burden as an immunotherapy biomarker: utility for the oncology clinic. Ann Oncol 2019;30:44-56.

7 Tsao MS, Kerr KM, Kockx M, et al. PD-L1 immunohistochemistry comparability study in real-life clinical samples: results of blueprint phase 2 project. J Thorac Oncol 2018;13:1302-11.

8 Udall M, Rizzo M, Kenny J, et al. PD-L1 diagnostic tests: a systematic literature review of scoring algorithms and test-validation metrics. Diagn Pathol 2018;13:12.

9 Hirsch FR, McElhinny A, Stanforth D, et al. PD-L1 immunohistochemistry assays for lung cancer: results from phase 1 of the blueprint PD-L1 IHC assay comparison project. J Thorac Oncol 2017;12:208-22.

10 Rimm DL, Han G, Taube JM, et al. A prospective, multi-institutional, Pathologist-Based assessment of 4 immunohistochemistry assays for PD-L1 expression in non-small cell lung cancer. JAMA Oncol 2017;3:1051-8.

11 Rimm DL. What brown cannot do for you. Nat Biotechnol 2006;24:914-6.

12 Liebler DC, Holzer TR, Haragan A, et al. Analysis of immune checkpoint drug targets and tumor Proteotypes in non-small cell lung cancer. Sci Rep 2020;10:9805.

13 Abubakar M, Figueroa J, Ali HR, et al. Combined quantitative measures of ER, PR, HER2, and KI67 provide more prognostic information than categorical combinations in luminal breast cancer. Mod Pathol 2019;32:1244-56.

14 Carvajal-Hausdorf DE, Schalper KA, Pusztai L, et al. Measurement of domain-specific HER2 (ErbB2) expression may classify benefit from trastuzumab in breast cancer. J Natl Cancer Inst 2015;107 doi:10.1093/jnci/djv136

15 Yardley DA, Kaufman PA, Huang W, et al. Quantitative measurement of HER2 expression in breast cancers: comparison with 'real-world' routine HER2 testing in a multicenter Collaborative Biomarker Study and correlation with overall survival. Breast Cancer Res 2015;17:41.

16 Wulfkuhle JD, Berg D, Wolff C, et al. Molecular analysis of HER2 signaling in human breast cancer by functional protein pathway activation mapping. Clin Cancer Res 2012;18:6426-35.

17 Jensen K, Erickson J, Webster S. Evaluation of tissue processing factors affecting HER2 IHC staining intensity in breast cancer cell lines. Cancer Res 2012;72.

18 Pierobon M, Silvestri A, Spira A, et al. Pilot phase I/II personalized therapy trial for metastatic colorectal cancer: evaluating the feasibility of protein pathway activation mapping for stratifying patients to therapy with imatinib and panitumumab. J Proteome Res 2014;13:2846-55.

19 Jameson GS, Petricoin EF, Sachdev J, et al. A pilot study utilizing multi-omic molecular profiling to find potential targets and select individualized treatments for patients with previously treated metastatic breast cancer. Breast Cancer Res Treat 2014;147:579-88.

20 Arnedos M, Vicier C, Loi S, et al. Precision medicine for metastatic breast cancer-limitations and solutions. Nat Rev Clin Oncol 2015;12:693-704.

21 Wulfkuhle JD, Yau C, Wolf DM, et al. Evaluation of the HER/PI3K/ AKT family signaling network as a predictive biomarker of pathologic complete response for patients with breast cancer treated with neratinib in the I-SPY 2 trial. JCO Precis Oncol 2018;2:1-20. doi:10.1200/PO.18.00024

22 Baldelli E, Bellezza G, Haura EB, et al. Functional signaling pathway analysis of lung adenocarcinomas identifies novel therapeutic targets for KRAS mutant tumors. Oncotarget 2015;6:32368-79.

23 Zupa A, Improta G, Silvestri A, et al. A pilot characterization of human lung NSCLC by protein pathway activation mapping. J Thorac Oncol 2012;7:1755-66.

24 Eisenhauer EA, Therasse P, Bogaerts J, et al. New response evaluation criteria in solid tumours: revised RECIST guideline (version 1.1). Eur J Cancer 2009;45:228-47.

25 Sereni MI, Baldelli E, Gambara G, et al. Kinase-driven metabolic signalling as a predictor of response to carboplatin-paclitaxel adjuvant treatment in advanced ovarian cancers. Br J Cancer 2017:117:494-502

26 Nanda R, Liu MC, Yau C, et al. Effect of pembrolizumab plus neoadjuvant chemotherapy on pathologic complete response in women with early-stage breast cancer: an analysis of the ongoing phase 2 adaptively randomized I-SPY2 trial. JAMA Oncol 2020;6:676-84

27 Abu-Khalaf MM, Hatzis C, Hodge KA, et al. Multiomic advanced diagnostics for CDK 4/6 drug target activation mapping of $\mathrm{HR+/}$ HER2- metastatic breast cancer [abstract]. 
28 Improta G, Zupa A, Fillmore $\mathrm{H}$, et al. Protein pathway activation mapping of brain metastasis from lung and breast cancers reveals organ type specific drug target activation. $J$ Proteome Res 2011:10:3089-97.

29 Signore M, Manganelli V, Hodge A. Antibody validation by Western blotting. Methods Mol Biol 2017;1606:51-70.

30 Baldelli E, Haura EB, Crinò L, et al. Impact of upfront cellular enrichment by laser capture microdissection on protein and phosphoprotein drug target signaling activation measurements in human lung cancer: implications for personalized medicine. Proteomics Clin Appl 2015;9:928-37.

31 Espina V, Wulfkuhle JD, Calvert VS, et al. Laser-capture microdissection. Nat Protoc 2006;1:586-603.

32 Baldelli E, Calvert V, Hodge A, et al. Reverse phase protein microarrays. Methods Mol Biol 2017:1606:149-69.

33 Pierobon M, Ramos C, Wong S, et al. Enrichment of PI3K-AKT-mTOR pathway activation in hepatic metastases from breast cancer. Clin Cancer Res 2017;23:4919-28.

34 Pin E, Federici G, Petricoin EF. Preparation and use of reverse protein microarrays. Curr Protoc Protein Sci 2014;75:27.7.1-9.

35 Mandarano M, Bellezza G, Belladonna ML, et al. Assessment of TILs, IDO-1, and PD-L1 in resected non-small cell lung cancer: an immunohistochemical study with clinicopathological and prognostic implications. Virchows Arch 2019;474:159-68.

36 Parra ER, Villalobos P, Mino B, et al. Comparison of different antibody clones for immunohistochemistry detection of programmed cell death ligand 1 (PD-L1) on non-small cell lung carcinoma. Appl Immunohistochem Mol Morphol 2018;26:83-93.

37 Lawson NL, Dix Cl, Scorer PW, et al. Mapping the binding sites of antibodies utilized in programmed cell death ligand-1 predictive immunohistochemical assays for use with immuno-oncology therapies. Mod Pathol 2020;33:518-30.

38 Atsaves V, Tsesmetzis N, Chioureas D, et al. PD-L1 is commonly expressed and transcriptionally regulated by STAT3 and MYC in ALKnegative anaplastic large-cell lymphoma. Leukemia 2017;31:1633-7.

39 Gown AM. Diagnostic immunohistochemistry: what can go wrong and how to prevent it. Arch Pathol Lab Med 2016;140:893-8.

40 Nixon AB, Schalper KA, Jacobs I, et al. Peripheral immune-based biomarkers in cancer immunotherapy: can we realize their predictive potential? J Immunother Cancer 2019;7:325.

41 Pin E, Stratton S, Belluco C, et al. A pilot study exploring the molecular architecture of the tumor microenvironment in human prostate cancer using laser capture microdissection and reverse phase protein microarray. Mol Oncol 2016;10:1585-94.

42 Diggs LP, Hsueh EC. Utility of PD-L1 immunohistochemistry assays for predicting PD-1/PD-L1 inhibitor response. Biomark Res $2017 ; 5: 12$
43 Bogen SA, Vani K, Sompuram SR. Molecular mechanisms of antigen retrieval: antigen retrieval reverses steric interference caused by formalin-induced cross-links. Biotech Histochem 2009;84:207-15.

44 Ramos-Vara JA, Miller MA. When tissue antigens and antibodies get along: revisiting the technical aspects of immunohistochemistry-the red, brown, and blue technique. Vet Pathol 2014;51:42-87.

45 Adam J, Le Stang N, Rouquette I, et al. Multicenter harmonization study for PD-L1 IHC testing in non-small-cell lung cancer. Ann Oncol 2018;29:953-8.

46 Ratcliffe MJ, Sharpe A, Midha A, et al. Agreement between programmed cell death ligand-1 diagnostic assays across multiple protein expression cutoffs in non-small cell lung cancer. Clin Cancer Res 2017;23:3585-91.

47 Marchetti A, Barberis M, Franco R, et al. Multicenter comparison of 22C3 PharmDx (Agilent) and SP263 (Ventana) assays to test PD-L1 expression for NSCLC patients to be treated with immune checkpoint inhibitors. J Thorac Oncol 2017;12:1654-63.

48 McLaughlin J, Han G, Schalper KA, et al. Quantitative assessment of the heterogeneity of PD-L1 expression in non-small-cell lung cancer. JAMA Oncol 2016;2:46-54.

49 Gaule P, Smithy JW, Toki M, et al. A quantitative comparison of antibodies to programmed cell death 1 ligand 1. JAMA Oncol 2017;3:256-9.

50 Pinato DJ, Mauri FA, Spina P, et al. Clinical implications of heterogeneity in PD-L1 immunohistochemical detection in hepatocellular carcinoma: the Blueprint-HCC study. Br J Cancer 2019;120:1033-6.

51 Brunnström H, Johansson A, Westbom-Fremer S, et al. PD-L1 immunohistochemistry in clinical diagnostics of lung cancer: interpathologist variability is higher than assay variability. Mod Pathol 2017;30:1411-21.

52 Kintsler S, Cassataro MA, Drosch M, et al. Expression of programmed death ligand (PD-L1) in different tumors. Comparison of several current available antibody clones and antibody profiling. Ann Diagn Pathol 2019;41:24-37.

53 Orme JJ, Jazieh KA, Xie T, et al. ADAM10 and ADAM17 cleave PD-L1 to mediate PD-(L)1 inhibitor resistance. Oncoimmunology 2020;9:1744980.

54 Romero Y, Wise R, Zolkiewska A. Proteolytic processing of PD-L1 by ADAM proteases in breast cancer cells. Cancer Immunol Immunother 2020;69:43-55

55 Wolf DM, Yau C, Wulfkuhle J, et al. Mechanism of action biomarkers predicting response to AKT inhibition in the I-SPY 2 breast cancer trial. NPJ Breast Cancer 2020;6:48.

56 Pierobon M, Robert NJ, Northfelt DW, et al. Multi-omic profiling of metastatic lesions to guide treatment selection: the side out 2 trial experience. JCO 2018;36:1077. 